\title{
Formación de pensamiento crítico en estudiantes de física de educación superior proyecto
}

Formation of critical thinking in students of physics of higher education

Ignacio Laiton Poveda

Escuela Teanológica Instituto Técnico Central. ilaiton@gmail.com

\section{Resumen}

El presente artículo pretende dar cuenta del proyecto de investigación "La solución de Problemas en ambientes simulados, para el desarrollo del pensamiento crítico" desarrollado como investigación dentro de la maestría en docencia de la Universidad de la Salle, y que proyecta ser un modelo a ser aplicado en contextos universitarios, no sólo dentro del ámbito antes citado, sino como aporte en el quehacer docente universitario y a la vez proyección de la enseñanza de las ciencias en nuestro país. En primer lugar se describe, enmarca y justifica el proyectoo, en segundo lugar se describe la forma en la que el autor pretende desarrollarlo, teniendo en cuenta su aplicación en las aulas de la Escuela Tecnológica Instituto Técnico Central.

\section{Palabras claves}

Pensamiento crítico, simulación, educación, autonomía.

\section{Abstract}

The present article seeks to give account of the developed investigation project as proposal of investigation inside the "Maestria en Docencia" of the University of La Salle. Seeks to be a model to be applied in university contexts, not just inside the above-cited environment, but like a contribution in the university educational chore and at the same time projection of the teaching of the sciences in our country. In the first place it is described, it frames and it justifies the project, in second place the form is described in the one that is sought to develop, keeping in mind its application in the dassroom of the Escuela Tecnológica Instituto Técnico Central

\section{Keywords}

Critical thinking, simulation, education, autonomy.

\section{Introducción y generalidades}

El presente artículo, pretende ser un aporte adicional al componente que, a pensar del autor, es el más importante en todas las instituciones que se precien de impartir una educación de calidad, en particular en la Escuela Tecnológica Instituto Técnico Central, que no es otro que el componente pedagógico. Pretende además ser complementario y enriquecedor de la posible discusión que sobre el tema debe darse en las instituciones de nivel universitario, cotejando y analizando otros aportes 
muy importantes que docentes prestantes han querido manifestar, no solo en nuestro quehacer cotidiano sino también a través de las páginas de diversas publicaciones.

En primer lugar, vale la pena destacar que la presente investigación pretende ser, y de esa manera debe verse en este artículo, una propuesta pedagógica para la enseñanza de las ciencias, en este caso en particular, la Física.

El pensamiento crítico

El pensamiento crítico, en su manera más general, puede ser definido por cualquier persona, de manera que, si abordamos a cualquier individuo, ciudadano del común o un docente de cualquier nivel de educación, seguramente encontraremos, en primer lugar, que estará de acuerdo en que debe ser enseñado, y en segundo lugar, nos daría una definición o propuesta de definición sobre lo que significa para él, el pensamiento crítico. Casi todos los docentes estaremos de acuerdo en que es deseable encontrar en nuestras aulas de dase, estudiantes críticos, pensantes, que analicen, que pregunten e indaguen sobre el conocimiento. Sin embargo surge aquí un primer cuestionamiento en nuestro camino hacia el pensamiento crítico: ¿son nuestros estudiantes el fruto de una educación media, básica y primaria, que los instruyó y educó en esas características que nosotros buscamos en ellos? Y seguramente un segundo: ¿Tenemos claro de qué hablamos cuando nos referimos al pensamiento crítico, y cómo se debe enseñar? Respuestas que cada uno de nosotros tiene y que seguramente entraríamos en una disertación con diferentes posidiones al respecto.

Sin pretender ser recurrente y repetitivo, respecto a aquellos discursos en donde se nos presenta una propuesta como "contraria a la educación tradicional, bancaria, memorística, transmisionista, etc." Muy común en todo este tipo de documentos, debemos decir que el pensamiento cítico, comienza a constituirse en una propuesta interesante en los diferentes países de Latinoamérica, como por ejemplo México, Costa Rica, en donde se han desarrollado propuestas en tal sentido, sin excluir que en Colombia hay algunos grupos preocupados y estudiosos del tema.

Lo interesante de la propuesta radica fundamentalmente en el hecho de que aplicarla en la práctica, implica desde el comienzo un cambio radical en su puesta en escena. La forma misma de asumir el ejercicio pedagógico diario de enfrentar un auditorio de estudiantes ansiosos de conocimientos, llámese "dictar una dase", ya no es igual, en el sentido dásico de la palabra. No es, entonces, una mera modificación teórica o de forma, se considera una profunda reflexión y puesta en práctica, podemos arriesgamos, por que no, a afirmar que no es posible asumir la enseñanza del pensamiento crítico, bajo la metodología dásica de enseñanza.

Se resalta la anterior característica, por el hecho, ya conocido, de las modas que llegan cada intervalo de tiempo sobre diversas tendencias y modelos pedagógicos, muy de moda cuando de elegir tal criterio se trataba, para ser plasmado en el Proyecto Educativo Institucional (PEI) de cada institución. Sin embargo, diversos y recientes estudios han demostrado que en un altísimo porcentaje la dase de los docentes sigue siendo la tradicional, tan criticada. Las nuevas tecnologías de la información y las comunicaciones (TICs) en la educación, también tratan de aportar en este aspecto, sin embargo, y en particular en nuestro entomo próximo, distan mucho de estar al alcance de todos nuestros estudiantes.

¿Y qué es el pensamiento crítico?

Diversos autores, entre los que se destacan Ennis, Facione, y el mismo Dewey, coinciden en resaltar que el pensamiento crítico se erige como necesario en nuestro mundo actual. Induso Boisvert (2004) señala que se destaca la importancia de facilitar a los estudiantes los medios para protegerse de manipulaciones y explotaciones, así como de vaivenes políticos. Y es en este sentido, que entre los múltiples propósitos que animarían a los profesores universitarios en su quehacer diario con el objetivo de refundar o recrear la profesión docente, es renovar su interés por su 
propia profesión. Una forma es la de generar, o al menos proponer, cambios educativos en las diversas instituciones universitarias encaminados a fomentar en los estudiantes capacidades tales como "aprender a aprender" en las diversas áreas y asignaturas. La necesidad de los estudiantes para que no solo adquieran un conocimiento ya elaborado que constituye la cultura y la ciencia de nuestra sociedad (Pozo, 1994), sino que también y de manera particularmente especial que adquieran habilidades y estrategias que les permitan aprender por si mismos nuevos conocimientos. El estudiante, sin embargo, encuentra que la academia no se los da, de tal forma que cuando arriban a su primer semestre universitario carecen del arsenal necesario para acometer dicha tarea.

Un intento de definición

Aun cuando no hay una definición unánime y univoca de los que se denomina "pensamiento crítico", comienza a verse en la literatura cierta unidad de terminologías en cuanto a las características esperadas en los sujetos con dicha forma de pensamiento, lo que facilita el ir agrupándolos, y a la vez ir identificando grupos de trabajo que daramente elaboran teorías firmes sobre el tema. Iniciaremos nuestra indagación sobre el tema citando algunos autores que se consideran importantes dentro de la construcción de la definición de pensamiento crítico.

Dewey (2007), define el pensamiento reflexivo como: "La consideración activa, persistente $y$ auidadosa de una creencia o forma supuesta de conocimiento a la luz de los fundamentos que la apoya y de las condusiones a las que tiende".

Ennis y Norris plantean que pensar críticamente es: "Un pensamiento razonado y reflexivo, orientado a una decisión de que creer o hacer" (Norris, 1989, citado por Boisvert, 2004). Paul (1992) plantea que "el pensamiento crítico es disciplinado $y$ autodinigido, $y$ ejemplifica las perfecciones del pensar adecuado ante un modo o área particular disciplinar"entiende mejor como la habilidad de los sujetos para hacerse cargo de su propio pensamiento. Esto requiere que desarrollen criterios y estándares apropiados para analizar y evaluar su propio pensamiento y utilizar rutinariamente esos criterios y estándares para mejorar su calidad.

López Calva (1998) describe que el pensamiento crítico es un pensamiento ordenado y daro, que lleva al conocimiento de la realidad por medio de la afirmación de juicios de verdad. En este aspecto resulta importante recordar que Lonergan (López, 1998) destaca que los tres primeros niveles de la estructura dinámica del conocimiento humano son "atender, entender y juzgar", enmarcando al pensamiento cútico en aquel tercer nivel. A su vez el autor destaca ciertas características del pensamiento crítico, comunes en varios de los autores arriba citados:

$>$ Analizar el valor de una afirmación.

$>$ Clasificar y categorizar.

$>$ Desarrollar conceptos.

$>$ Formular explicaciones.

$>$ Dar razones.

Buscar falacias.

Resolver problemas.

\section{Desarrollo: la propuesta}

A partir de las caracterizaciones que los autores han propuesto, Boisvert (2004) ha resumido y estudiado de manera detallada tales propuestas, llegando a algunas consideraciones prácticas, que, aunque abarcan demasiados ítems para ser tratados en una sola investigación en el aula, también expone la posibilidad de ser adaptada a cualquier contexto, eligiendo de acuerdo al mismo, las características que a su juicio el investigador considere más adecuadas. En este sentido, y basados en la definición y caracterización enunciada por Jacques Boisvert, Docente de la Universidad De 
Quebec - Canadá se ha elegido para su implementación en el proyecto con estudiantes de la Escuela Teanológica los siguientes ariterios a manera de las autro capacidades del pensamiento crítico que un estudiante de primeros semestres debe poseer:

Evaluación de la credibilidad de una fuente.

$>$ Análisis de argumentos.

$>$ Presentación de una postura con ayuda de una argumentación oral o escrita.

$>$ Respetar etapas del proceso en la resolución de problemas.

Se considera que las anteriores quatro habilidades, se complementan y hacen que el proyecto tenga coherencia al momento de su aplicación. El autor que lo fundamenta recomienda tener en cuenta aspectos como: la aplicación freauente por parte del estudiante en su contexto, la aplicación en la vida real cotidiana del individuo, la exigencia de aplicar conocimientos previos, y el enfrentarse a la solución de situaciones problemáticas.

Evaluación de la credibilidad de una fuente

La evaluación de la credibilidad de una fuente se hace fundamental, en nuestra época, en donde es tan frecuente que el estudiante, al ser colocado en la posición de consulta, acude casi ineludiblemente a Internet, encontrando a su disposición multitud de información, información que en la mayoría de los casos, se asume verdadera, sin que se increpe sobre su veracidad, su origen o la verdadera capacidad del escritor. En este sentido dentro del proyecto se propone al estudiante la consulta de varias fuentes de información sobre el tema a estudiar (cinemática, por ejemplo), cada una con un perfil diferente, en este caso los textos de Richard Feynman, de Serway y el texto de Quiroga para el bachillerato, a los que se le pide realice una lectura, y contraste a través de una matriz comparativa o cuadro comparativo, teniendo en consideración criterios tales como reputación del autor, lenguaje utilizado, formulación matemática del autor, entre otros.

Análisis de argumentos

En cuanto al análisis de argumentos, se pretende que el estudiante al acceder a un documento de cualquier índole, en este caso científico, identifique los argumentos, premisas y condusiones que el autor pretende plasmar en el escrito. En particular se tendrán en cuenta criterios de reconocimiento de conclusiones, identificación de los argumentos sobre los que el autor se apoya y el descubrir la estructura de la argumentación. En este caso se le proporciona al estudiante un texto de divulgación científica relacionado con el tema estudiado, ante el cual se le solicita explicitar las ideas principales del autor, las condusiones a las que llega y se pide la elaboración de un cuadro, resumen o mapa conceptual que rebele la estructura del escrito.

Presentación de una postura con ayuda de una argumentación oral o escrita

Un tercer ítem corresponde a la capacidad de asumir una posición argumentada frente a un hecho o información, aspecto en el cual se manifiesta explícitamente la actuación del estudiante, ya que lo expone o lo insita a escribir o a hablar sobre su propio raciocinio, tomando con claridad una posición frente a los hechos. En la investigación se consideran criterios de; asumir una tesis, presentar razones, aportar adaraciones y considerar variantes o posibilidades que da el tema.

Para nuestro caso se le proporciona al estudiante un texto previamente elegido de Física, frente al cual debe manifestar una opinión con una posición dara, haciendo uso de un lenguaje coherente con su nivel universitario y manejando terminología científica acorde.

Respetar el proceso en la solución de problemas

Finalmente, y constituyéndose en aspecto fundamental, la correcta aplicación de un proceso en la solución de problemas, será nuestro ítem primordial, en la enseñanza de la física se suelen enfrentar situaciones problémicas que exigen del estudiante el tener un plan de trabajo sin el aual los resultados serán obtenidos a medias o simplemente no se obtendrá ningún resultado. A pesar de que muchos autores han escrito sobre solución de problemas, para la presente investigación se 
tendrán en cuenta los aspectos de: definición adecuada del problema, formulación de posibles caminos de solución, dando importancia a la existencia de varios de ellos, evaluación de las soluciones formuladas y finalmente la puesta en practica de tales soluciones, obteniendo en lo posible la respuesta ideal al problema.

Un aspecto fundamental en este ítem, consiste en el uso de simuladores gratuitos tomados de INTERNET, o del programa Interactive Physics, para simular la situación problémica planteada, contrastando los resultados teóricos con lo observado en el simulador.

El proyecto se desarrolla con una metodología mixta cualitativa - cuantitativa, con un diseño de series cronológicas, con $\mathbf{3 0}$ estudiantes del curso de Física I de la Escuela Tecnológica Instituto Técnico Central de la carrera de Electromecánica, teniendo aplicación del pretest, tres momentos de situación problemática, aplicación de test al final de cada situación y aplicación de pos test y entrevistas a estudiantes, como se observa en la figura 1.

Figura 1. Actividades a realizar en el proyecto

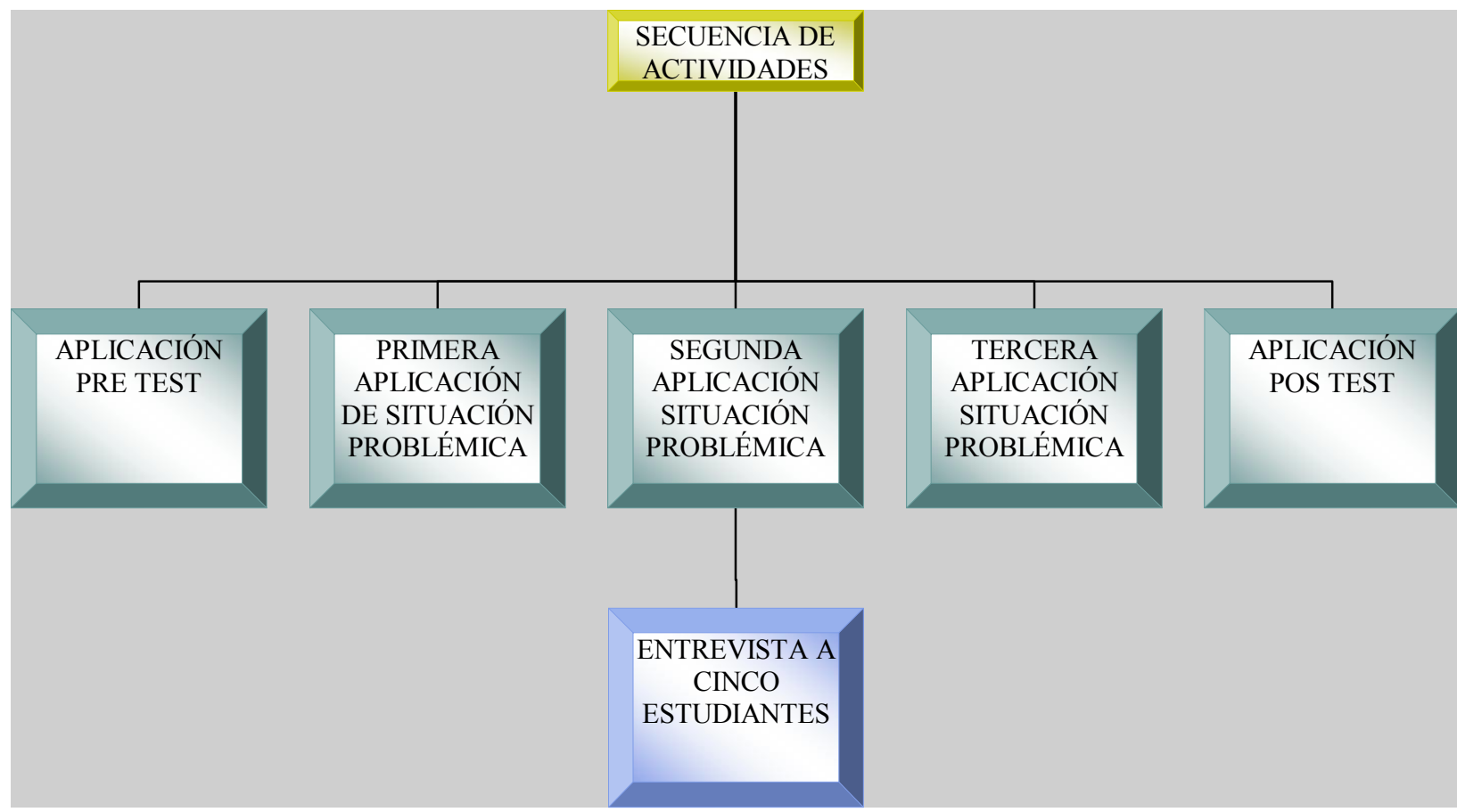

\section{Avances y conclusiones}

Actualmente el proyecto ha desarrollado su parte inicial de dos etapas, que corresponde a la identificación de las capacidades del pensador crítico presentes en los estudiantes de primeros semestres de Ingeniería. Se ha aplicado a la población objetivo el pretest correspondiente y la primera situación problémica correspondiente al lanzamiento parabólico, para lo aual cada estudiante eligió una situación a resolver dentro de los diferentes deportes, por ejemplo futbol, baloncesto, etc. Asignando parámetros reales de las dimensiones de los espacios para cada deporte, y realizando una consulta con evaluación de fuentes, planteamiento del problema, solución argumentada, toma de posición frente al problema, y finalmente la simulación, confrontando resultados.

Para cada una de las cuatro capacidades de pensamiento crítico, se eligieron tres criterios que convierten en cuantitativos nuestros datos, asignando un valor de $\mathbf{2}$ para el cumplimiento del 
criterio, 1 para la existencia parcial del criterio y 0 para la no-existencia del mismo. Se tienen los siguientes citerios para cada habilidad:

$>$ Evaluación de la credibilidad de una fuente.

a. Experiencia y reputación del autor

b. Acuerdo con otras fuentes, formulación matemática y nivel del texto.

c. Capacidad de dar razones argumentativas, claridad en las explicaciones y ejemplos.

$>$ Evaluación de análisis de argumentos.

a. Reconocimiento de conclusiones

b. Identificación de razones y argumentos principales del autor.

c. Explicar estructura de la argumentación.

> Postura con argumentación escrita

a. Emplea el vocabulario adecuado de acuerdo a su nivel.

b. Se identifica con claridad su postura.

c. Expone elementos argumentativos.

$>$ Aplicación del proceso de resolución de problemas.

a. Identifica con claridad los elementos que componen el problema.

b. Formula un conjunto de soluciones o parámetros.

c. Pone en práctica la solución, diseñándola en el simulador de manera adecuada.

Después de la aplicación del pretest, se obtuvieron los siguientes resultados resumidos en la siguiente tabla.

\begin{tabular}{|c|c|c|c|c|c|c|c|c|c|c|c|c|c|}
\hline \multicolumn{14}{|c|}{ TABLA DE EVALUACION HABILIDADES DE PENSAMIENTO CRITICO PRETEST } \\
\hline \multirow[b]{2}{*}{ PERSONA } & \multicolumn{3}{|c|}{ HABILIDAD 1} & \multicolumn{3}{|c|}{ HABILIDAD 2} & \multicolumn{3}{|c|}{ HABILIDAD 3} & \multicolumn{3}{|c|}{ HABILIDAD 4} & \multirow[b]{2}{*}{ TOTAL } \\
\hline & C1 & $\mathbf{C 2}$ & $\mathbf{C 3}$ & C1 & C2 & $\mathbf{C 3}$ & C1 & $\mathbf{C 2}$ & C3 & C1 & $\mathbf{C 2}$ & C3 & \\
\hline 1 & $\mathbf{0}$ & $\mathbf{0}$ & $\mathbf{0}$ & $\mathbf{0}$ & 2 & $\mathbf{0}$ & $\mathbf{0}$ & $\mathbf{0}$ & $\mathbf{0}$ & $\mathbf{0}$ & $\mathbf{0}$ & $\mathbf{0}$ & 2 \\
\hline 2 & $\mathbf{0}$ & 2 & 0 & 1 & 1 & 1 & 1 & 1 & 0 & 1 & 1 & 1 & 10 \\
\hline 3 & 1 & 0 & 0 & 0 & 0 & 0 & 0 & 1 & 0 & 0 & 0 & 0 & 2 \\
\hline 4 & 0 & 1 & 0 & 0 & 0 & 1 & 0 & 0 & 0 & 0 & 0 & 0 & 2 \\
\hline 5 & 2 & 1 & 0 & 0 & 0 & 0 & 1 & 1 & 0 & 0 & 0 & 0 & 5 \\
\hline 6 & 1 & 1 & 0 & 0 & 0 & 1 & 0 & 0 & 0 & 0 & 0 & 1 & 4 \\
\hline 7 & 1 & 0 & 0 & 1 & 1 & 1 & 2 & 2 & 1 & 0 & 1 & 0 & 10 \\
\hline 8 & 1 & 0 & 0 & 0 & 0 & 0 & 1 & 2 & 0 & 1 & 0 & 0 & 5 \\
\hline 9 & 1 & 0 & 0 & 1 & 0 & 0 & 1 & 1 & 0 & 0 & 1 & 0 & 5 \\
\hline 10 & 1 & 0 & 1 & 0 & 1 & 0 & 1 & 0 & 0 & 1 & 0 & 0 & 5 \\
\hline 11 & 1 & 1 & 1 & 0 & 0 & 0 & 1 & 0 & 0 & 2 & 1 & 1 & 8 \\
\hline 12 & 0 & 1 & 1 & 1 & 0 & 0 & 1 & 2 & 1 & 0 & 1 & 1 & 9 \\
\hline 13 & 0 & 0 & 1 & 2 & 0 & 1 & 1 & 1 & 0 & 2 & 2 & 1 & 11 \\
\hline 14 & 0 & 2 & 0 & 0 & 0 & 1 & 1 & 0 & 0 & 0 & 1 & 1 & 6 \\
\hline 15 & 0 & 0 & 1 & 2 & 2 & 0 & 1 & 1 & 1 & 1 & 2 & 0 & 11 \\
\hline 16 & 1 & 0 & 0 & 0 & 2 & 2 & 0 & 1 & 0 & 0 & 1 & 1 & 8 \\
\hline 17 & 0 & 0 & 1 & 1 & 0 & 0 & 1 & 0 & 0 & 2 & 1 & 0 & 6 \\
\hline 18 & 0 & 0 & 0 & 0 & 0 & 0 & 1 & 1 & 0 & 0 & 1 & 1 & 4 \\
\hline 19 & 1 & 1 & 0 & 1 & 0 & 1 & 2 & 2 & 2 & 0 & 1 & 1 & 12 \\
\hline 20 & 1 & 1 & 1 & 0 & 1 & 0 & 0 & 1 & 0 & 0 & 2 & 1 & 8 \\
\hline 21 & 2 & 0 & 0 & 1 & 0 & 0 & 2 & 2 & 2 & 1 & 1 & 0 & 11 \\
\hline 22 & 0 & 0 & 0 & 0 & 0 & 0 & 1 & 2 & 2 & 1 & 1 & 0 & 7 \\
\hline 23 & 0 & 0 & 0 & 1 & 0 & 1 & 0 & 0 & 0 & 0 & 1 & 0 & 3 \\
\hline 24 & 0 & 0 & 0 & 0 & 0 & 0 & 2 & 1 & 2 & 2 & 1 & 1 & 9 \\
\hline 25 & 1 & 1 & 0 & 1 & 0 & 1 & 1 & 1 & 0 & 1 & 1 & 0 & 8 \\
\hline \multicolumn{14}{|l|}{26} \\
\hline 27 & & & & & & & & & & & & & \\
\hline TOTAL & 15 & 12 & 7 & 13 & 10 & 11 & 22 & 23 & 11 & 15 & 21 & 11 & 171 \\
\hline Promedios & 0.6 & 0.48 & 0.28 & 0.52 & 0.4 & 0.44 & 0.88 & 0.92 & 0.44 & 0.6 & 0.84 & 0.44 & 6,84 \\
\hline
\end{tabular}

Tabla No. 1. Resultados aplicación pretest 
Se observa que los resultados de esta aplicación revelan un bajo promedio en la evaluación de las habilidades de pensamiento crítico, ya que sus promedios individuales nunca superan ni siquiera al $50 \%$ de la nota máxima, en este caso 2 Mientras que en el promedio grupal solo se obtiene un 6,84 sobre un total de 24 como nota máxima. Se revela entonces después del análisis estadístico, que por espacio no se induye aquí, que la deficiencia en las habilidades de pensamiento crítico en los estudiantes de la Escuela Teanológica Instituto Técnico Central, es bastante grande, lo aual valida la intervención que se realiza en el grupo a través de la metodología antes descrita.

Se considera que al aplicar en el aula las habilidades enunciadas por Boisvert (2004), a manera de compendio de lo que varios autores proponen, obtendremos estudiantes más capaces de aprender a aprender, de indagar, de cuestionar, de argumentar adecuadamente, y en últimas de adquirir habilidades que les permitan desempeñarse de mejor manera en el mundo actual, que les exige cada día más poder de adaptación y aprendizaje de nuevas tecnologías, en el mundo globalizado. Es importante finalmente recalcar que, los anteriores criterios se aplican dentro del contexto de la enseñanza de la Física, pensándola como generadora de pensamiento critico, dándole importancia a la parte conceptual de la misma, que requiere, como cualquier otro texto, de interpretación, análisis y aplicación en la resolución de problemas. Solo nos queda entonces, a pesar del cambio que esto implica, ponerlo en práctica y esperar que los resultados sean los ideales, de tal modo que al final de las autro aplicaciones encontremos resultados alentadores sobre el pensamiento crítico de nuestros estudiantes.

\section{Bibliografía}

Boisvert J. (2004). La formación del pensamiento crítica. México Fondo de cultura económica.

Dewey, J. (2007). ¿Cómo pensamos?, relación entre pensamiento reflexivo y proceso educativo. Espana, Paidós.

López Calva, M. (1998). Pensamiento Crítico y creatividad en el aula. México, Trillas.

Pozo, J. (1994). La Solución de Problemas. España Santillana,a

Paul, R. (1992). "Critical thinking: What, Why and How'New directions for Community colleges. 